\title{
Estudo transversal sobre uso de ferramentas virtuais para orientar a atividade física durante a COVID-19
}

\section{Cross-sectional study on the use of virtual tools to guide physical activity practice during COVID-19}

\section{AUTORES \\ Jean Augusto Coelho Guimarães ${ }^{1}$ (D) \\ Paulo Henrique Guerra ${ }^{2,3}$ (D) \\ Deisy Terumi Ueno ${ }^{1}$ (D) \\ Ana Elisa Messetti Christofoletti ${ }^{1}$ (D) \\ Priscila Missaki Nakamuraa ${ }^{1,4}$ (D) \\ 1 Universidade Estadual Paulista. Instituto de Biociências. Programa de Pós-Graduação em Ciências da Motricidade, Departamento de Educação Física. Rio Claro, São Paulo. Brasil. \\ 2 Universidade Federal da Fronteira Sul. Chapecó, Santa Catarina. Brasil. \\ 3 Grupo de Estudos e Pesquisas Epidemiológicas em Atividade Física e Saúde da Universidade de São Paulo, São Paulo, São Paulo, Brasil. \\ 4 Instituto Federal de Educação, Ciência e Tecnologia do Sul de Minas Gerais. Muzambinho, Minas Gerais, Brasil.}

\section{CONTATO}

Jean Augusto Coelho Guimarães

jean13ef@gmail.com

Av. 24A, n. 1515, Bela Vista - Rio Claro, São

Paulo, Brasil.

CEP: 13506-900.

DOI

$10.12820 /$ rbafs. $25 \mathrm{e} 0150$

\section{(cc) BY}

Este trabalho está licenciado com uma Licença Creative Commons - Atribuição 4.0 Internacional.

\begin{abstract}
RESUMO
A elevada taxa de transmissão e mortalidade pela COVID-19 acarretou o fechamento de centros de movimentação social, dentre eles locais para prática de atividade física. Com isso, o objetivo do estudo foi identificar as principais estratégias e ferramentas tecnológicas virtuais, percepção dos profissionais de educação física (PEF) acerca das adaptações, pontos positivos, negativos e feedbacks dos clientes para orientar a prática de atividade física durante o isolamento social/restrição de circulação devido ao vírus SARS-COV-2. Optou-se pela pesquisa transversal, quanti-qualitativa, com técnica de amostragem "bola de neve", sendo recrutados PEF das cinco regiões do Brasil. Os participantes responderam um questionário online com questões sociodemográficas, uso das ferramentas tecnológicas virtuais, perfil dos clientes e questões discursivas acerca da orientação online à atividade física. Foi realizada estatística descritiva e análise por codificação simples. Participaram $110 \mathrm{PEF}$ de 65 cidades do país, os quais geralmente atendem em academias de ginástica $(59,8 \%)$ e ao ar livre (34,5\%), tendo clientes adultos $(92,9 \%)$, do sexo feminino $(51,8 \%)$. Dentre as ferramentas tecnológicas virtuais utilizadas estão "vídeos" (60,9\%) e "Whatsapp web" (60,0\%), geralmente ferramentas gratuitas $(67,5 \%)$ e, para que o trabalho ocorra, são necessárias adaptações estruturais/materiais e profissionais. Embora haja aspectos negativos e dificuldades na orientação remota à prática de atividade física, os aspectos positivos e facilidades permitem que o $\mathrm{PEF}$ se conecte com seus respectivos clientes mesmo durante a pandemia, estimulando e, orientando de forma segura, a prática de atividade física. Esta temática necessita ser mais explorada para que essas ferramentas auxiliem os PEF.
\end{abstract}

Palavras-chave: Atividade física; Educação física; Pandemia; Serviços online; Brasil.

\section{ABSTRACT}

High rate of transmission and mortality by COVID-19 resulted in the closure of social movement centers, including places to physical activity practice (PA). Thus, the aim of the study was to identify the main technological strategies and tools, perception of physical education professionals (PEP) about the adaptations, positives, negatives and feedbacks of clients to guide physical activity practice during social isolation/circulation restriction due to the $S A R S-C O V-2$ virus. It was opted for cross-sectional, quanti-qualitative research, using the "snowball" sampling technique and PEP was recruited from the five regions of Brazil. Participants answered an online questionnaire with sociodemographic questions, use of virtual technological tools, students/clients profiles and discursive questions about online orientation to physical activity. Descriptive statistics and analysis by simple coding were performed. 110 PEP from 65 cities in the country participated, which generally serve in gyms (59.8\%) and outdoors (34.5\%), with adult students/clients (92.9\%), female (51.8\%). Among the virtual technological tools used are "videos" (60.9\%) and "Whatsapp web" (60.0\%), generally free tools (67.5\%) and, for the work to occur, professionals and structural/material adaptations are required. Although there are negative aspects and difficulties in remote guidance to physical activity practice, the positive aspects and facilities allow the PEP to connect with its respective students/clients even during the pandemic, stimulating and securely guiding to physical activity practice. This theme needs to be further explored for these tools to assist PEP.

Keywords: Physical activity; Physical education; Pandemic; Online services; Brazil.

\section{Introdução}

Em março de 2020, a Organização Mundial de Saúde (OMS) declarou estado de pandemia devido à rápida transmissão e aumento da mortalidade causada pelo vírus SARS-COV-2, que é responsável pela doença denominada coronavírus (COVID-19). Esta doença causa uma síndrome respiratória aguda que já atingiu mais de 200 países ao redor do mundo, sendo que no dia 13 de outubro de 2020 já apresentava mais de 38 milhões de casos confirmados e mais de 1 milhão de mortes ${ }^{1}$. Nessa mesma data, o Brasil é o terceiro país com maior número de casos confirmados, atingindo 
5.094.979 pessoas e 150.488 mortes, ficando atrás somente dos Estados Unidos e da Índia 2 .

Ainda não dispondo de uma forma efetiva de imunização, foram recomendadas algumas estratégias coletivas para combater o contágio e a propagação do vírus, envolvendo além da higienização das mãos, uso de álcool gel e máscara, a adoção de medidas de distanciamento social e restrição de circulação em espaços públicos ${ }^{3}$. No Brasil, embora com características distintas entre as regiões e estados, o decreto 4.310/20 previu o fechamento de centros de movimentação social, como as academias de ginástica e clubes a partir do dia 18 de março.

Deste modo, diversas classes profissionais, como os Profissionais de Educação Física (PEF), ficaram impossibilitados de atuarem tanto em ambientes fechados, quanto em ambientes ao ar livre, devido ao risco de contágio e disseminação do vírus. As restrições quanto à atuação presencial dos $\mathrm{PEF}$ foram recomendadas pela OMS, Ministério da Saúde ${ }^{4}$ e algumas organizações científicas da área, como a Sociedade Brasileira de Atividade Física e Saúde, Colégio Brasileiro de Medicina do Esporte e Sociedade Brasileira de Medicina do Exercício e do Esporte. Ainda, pesquisadores nacionais levantaram a reflexão de que embora seja reconhecida a importância da prática de atividade física para a saúde e o impacto econômico para o setor, reabrir os serviços neste momento não seria recomendado pelo elevado risco à saúde, principalmente para populações mais vulneráveis, como pessoas com doenças crônicas e idosos ${ }^{5}$.

Assim, para que esses profissionais continuassem orientando à prática de atividades e exercícios físicos de seus alunos/clientes, de forma segura, uma das estratégias foi migrar as aulas e acompanhamentos para as plataformas digitais. Estratégias estas que foram estimuladas pela OMS e o American College of Sports Medicine (ACSM), que divulgaram em seus sites alguns materiais auxiliares como sites, vídeos e leituras confiáveis ${ }^{6,7}$ incentivando a prática de atividade física e redução do comportamento sedentário. Chen et al. ${ }^{8}$ apontaram que, nesse período de distanciamento social, o acesso aos profissionais de forma online, aplicativos e vídeos seguros pode ser uma alternativa para manter e estimular a prática de atividade física da população. Todavia, ainda não há informações sobre os aspectos desse formato de intervenção, principalmente durante a pandemia, por parte dos PEF e seus alunos/clientes.

Visto que a prática de atividade física expressa evidências positivas no controle e prevenção de doenças crônicas não transmissíveis, como diabetes, hiperten- são e câncer ${ }^{9}$, além de gerar benefícios aos aspectos biopsicossociais $^{10}$, ao sistema imunológico dos praticantes $^{11,12}$ e; reconhecendo o potencial dos meios digitais enquanto facilitador à orientação das práticas de atividade física durante a pandemia, o objetivo deste estudo foi identificar as principais estratégias e ferramentas tecnológicas virtuais mais utilizadas, assim como a percepção dos PEF acerca das adaptações, pontos positivos, negativos e feedbacks dos alunos, sobre a orientação à prática de atividade física no contexto do isolamento social/restrição de circulação, em virtude da pandemia causada pelo vírus SARS-COV-2.

\section{Método}

Esta pesquisa transversal, com abordagem mista para coleta e análise de dados, contendo elementos quantitativos e qualitativos foi aprovada pelo Comitê de Ética do Instituto Federal de Educação, Ciência e Tecnologia do Sul de Minas Gerais, nº. 4.108.506.

Para composição da amostra, optou-se pela técnica de amostragem "bola de neve". Método utilizado em pesquisas que se desconhecem os possíveis participantes ou não há capacidade de mensurá-los antecipadamente ${ }^{13}$, implicando em uma amostragem não probabilística, sendo formada durante o processo da própria coleta de dados. A amostra tem de expressar características em comum, que são o elo facilitador para a identificação dos posteriores participantes. Assim, ocorre de forma cadenciada, na qual o pesquisador solicita aos participantes para compartilharem, com os seus semelhantes, o convite à participação na pesquisa ${ }^{14,15}$.

O processo de recrutamento, composição e identificação da amostra ocorreu entre 30 de junho e 7 de agosto de 2020, por meio das redes sociais virtuais, para facilitar a participação de PEF de diferentes regiões do Brasil. Os critérios para a participação foram: a) Ter formação em Educação Física (Bacharelado ou Licenciatura); b) Ter vínculo com alunos durante o contexto do isolamento social/restrição de circulação na pandemia por COVID-19; e c) Utilizar as tecnologias virtuais como ferramenta facilitadora para prescrição/ orientação de programas de treinamento e aulas.

Foram considerados desistentes os participantes que não responderam todo o questionário ou que enviaram um e-mail ao pesquisador principal solicitando a desistência de participação na pesquisa. No corpo deste e-mail, o participante deveria informar o código que ele mesmo criou no questionário, de cinco a seis dígitos.

Para ampliar a disseminação do convite à pesquisa, 
o pesquisador principal contatou os Conselhos Federal e Regionais de Educação Física, solicitando auxílio para divulgá-la em âmbito nacional, por meio do e-mail dos associados e seus canais eletrônicos de divulgação. Ademais, na tentativa de manter ampliada a divulgação, foram identificados cinco pesquisadores "influenciadores" da área em cada região do Brasil, sendo Norte, Nordeste, Centro-Oeste, Sudeste e Sul (n = 25), que também foram convidados a auxiliarem na divulgação desta pesquisa em suas redes de contatos. Todas essas solicitações foram enviadas via e-mail, cujo link para acesso ao questionário online estava disponível. Ainda, houve divulgação da pesquisa e do link do questionário nas redes virtuais e aplicativos (Facebook, Instagram, Whatsapp) e, contatos dos respectivos autores desta pesquisa, além de que os respondentes também foram convidados a compartilhar a pesquisa com mais três a cinco profissionais de seu conhecimento. Entretanto, não houve controle de perdas.

Para a coleta de dados, foi construído um questionário, posteriormente avaliado pelos pesquisadores do presente estudo e mais quatro pós-graduandos com experiência em pesquisas mistas, membros do Núcleo de Atividade Física, Esporte e Saúde. Após os ajustes para melhor compreensão das questões, o questionário foi inserido dentro da plataforma Google Forms. Para os respondentes terem acesso às perguntas, eles deveriam inicialmente ler o Termo de Consentimento Livre e Esclarecido (TCLE) que estava inserido na seção 1 (um) na forma de figura e link, sendo que este último redirecionava o participante para o documento online no Google Drive, podendo ser baixado. Após a leitura completa do TCLE, o participante concordando com os termos deveria clicar na opção "aceito", acessando a seção 2 (dois) do questionário e das perguntas.

De forma anônima, os participantes responderam 11 questões que abordaram sexo (1-Masculino ou 2-Feminino), idade (descritiva), localidade por região (1-Nordeste, 2-Norte, 3-Centro-Oeste, 4-Sudeste ou 5-Sul), cidade de atuação (descritiva), nível de formação acadêmica (1-Graduação, 2-Especialização, 3-Mestrado ou Doutorado) e ano de conclusão (descritiva, com posterior categorização 1- Até 2000, 2- 2001 a 2010, 3- 2011 a 2020).

Também questionou-se sobre os locais de atuação profissional (1-Academias, 2-Clubes, 3-Ao ar livre, 4-Espaços personalizados e 5-Home Care/atendimento domiciliar), perfil dos respectivos alunos/clientes (1-Crianças, 2-Adolescentes, 3-Adultos, 4-Idosos e
5-Pessoas com necessidades especiais) e predominância quanto ao sexo (1-Homens, 2-Mulheres ou 3-Não há diferença); quais as ferramentas tecnológicas virtuais mais utilizadas (1-Aplicativos específicos, 2-Whatsapp web, 3-FaceTime, 4-Skype, 5-Zoom, 6-Google Meet, 7- Planilhas no Excel/Word, 8- Vídeos (gravados e disponibilizados pelo próprio profissional e 9- $\mathrm{Ou}-$ tros) e, se fossem selecionados aplicativos específicos ou outras ferramentas, foi pedido para citarem o nome (descritiva) e, por último, a forma de acesso (1-Paga, 2-Gratuita e 3-As duas possibilidades).

Foram adicionadas quatro questões abertas, com $o$ intuito de identificar a percepção dos PEF sobre as ferramentas mais utilizadas, sendo as principais adaptações realizadas para a atuação profissional durante a pandemia da COVID-19, os pontos positivos e negativos acerca da utilização das ferramentas e, os feedbacks mais reportados pelos alunos/clientes desses $\mathrm{PEF}$ quanto às adaptações realizadas e ao novo formato de orientação. Para melhor visualização, o questionário completo pode ser acessado no link: https://drive.google.com/file/d/1Xmlfdj9ZV6jxIN0ToS5oROA7pd$\mathrm{gBUW} /$ view? $\mathrm{usp}=$ sharing.

Para as variáveis quantitativas, foi realizada estatística descritiva e cálculo de prevalência para as questões objetivas, utilizando-se para tanto o programa SPSS 21.0. Para as questões discursivas, optou-se pela análise por codificação simples, a qual evidencia palavras e frases semelhantes redigidas pelos participantes ${ }^{16}$. A priori, foram criadas as seguintes categorias compondo a matriz conceitual: a) Principais adaptações, b) Pontos positivos, c) Pontos negativos, d) Feedbacks dos alunos. A matriz conceitual foi criada para auxiliar na identificação de pontos importantes e servir de guia durante a análise dos relatos dos participantes. Após isso, iniciaram-se as análises de codificação simples, realizadas manualmente por meio de dois avaliadores, para que os resultados fossem comparados. Durante esse processo de análise, houve a criação das subcategorias relacionadas às categorias principais: a) estruturais/materiais e profissionais, b) ferramentas/aplicativos, segurança e profissionais; c) estruturais/materiais, ferramentas/ aplicativos e profissionais e d) facilidades e dificuldades.

\section{Resultados}

Sem registros de desistências, participaram deste estudo $110 \mathrm{PEF}$, sendo $58,1 \%$ ( $\mathrm{n}=64$ ) do sexo masculino, com idade média de 34,4 \pm 9,8 anos, estando distribuídos pelas regiões Sudeste 52,7\% ( $=58)$, Sul 19,1\% (n 
= 21), Nordeste 14,5\% ( $\mathrm{n}=16)$, Norte 7,3\% $(\mathrm{n}=8)$ e Centro-Oeste 6,4\% (n= 7) do Brasil.

Ao todo, profissionais de 65 cidades responderam o questionário, sendo que a cidade de São Paulo obteve a maior prevalência de respondentes, $15,4 \% \operatorname{PEF}(n=10)$. Em relação ao nível de formação acadêmica, 40,0\% (n = 44) são graduados, $38,2 \%(n=42)$ especialistas e $21,8 \%$ $(n=22)$ mestres ou doutores, sendo que mais de 60,0\% concluíram seus cursos entre os anos de 2011 e 2020.

Acerca dos locais em que estes PEF geralmente atendem, destes 59,8 \% ( $\mathrm{n}=67)$ apontaram academias de ginásticas, $34,5 \%(\mathrm{n}=38)$ em locais ao ar livre, $27,7 \%(\mathrm{n}=31)$ em home care/atendimentos domiciliares, 25,9\% ( $n=29)$ em espaços personalizados (estúdios e clínicas que promovem atividade física) e 14,3\% $(n=14)$ em clubes. Os perfis de alunos mais atendidos por esses PEF foram adultos $92,9 \%(n=104)$ e idosos $57,1 \%(\mathrm{n}=64)$, do sexo feminino $51,8 \%(\mathrm{n}=58)$.

Durante a pandemia por COVID-19, dentre as ferramentas tecnológicas virtuais (Figura 1) que estes profissionais utilizaram, enquanto meio facilitador para orientar a prática de atividade física, foram citados os "vídeos gravados e disponibilizados pelo próprio profissional" 60,9\% (n = 67) e "Whatsapp Web" 60,0\% (n =66) como as mais frequentes. Além destes, também foram citados "Aplicativos específicos" 39,1\% ( $=43)$, sendo que o Mfit 29,2\% ( $\mathrm{n}=7)$ e Treinus 12,5\% ( $\mathrm{n}=$ 3) prevaleceram dentre 24 aplicativos relatados. Ainda na opção "Outros" 8,2\% ( $\mathrm{n}=9)$, os PEF citaram o "Instagram" 2,7\% ( $=3)$, "Youtube" 2,7\% ( $=3)$, "Site próprio" 1,8\% (n=2), "Formulários Google” 0,9\% (n =

\begin{tabular}{|c|c|c|c|}
\hline \multirow{3}{*}{ 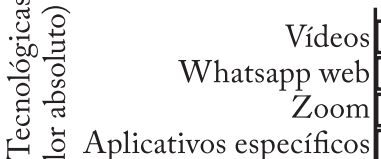 } & \multicolumn{3}{|c|}{35} \\
\hline & & 43 & 23 \\
\hline & 26 & 22 & \\
\hline$\stackrel{\oplus}{\oplus}$ Aplicativos específicos & 27 & 16 & \\
\hline$\approx$ I Planilhas Excel/Word & 29 & 12 & \\
\hline Google Meet & 35 & & \\
\hline Skype & $8] 2$ & & \\
\hline $\begin{array}{r}\text { Facetime } \\
\text { Outros }\end{array}$ & \begin{tabular}{c|c|}
5 & 4 \\
7 & \\
$y$
\end{tabular} & $\begin{array}{l}\square \mathrm{N} \\
\square \mathrm{F}\end{array}$ & $\begin{array}{l}\text { lino } \\
0\end{array}$ \\
\hline
\end{tabular}

Figura 1 - Frequência das ferramentas tecnológicas virtuais utilizadas pelos profissionais de educação física para orientar a prática de atividade física durante a pandemia da COVID-19, $2020(\mathrm{n}=110)$.

Quadro 1 - Seleção dos principais relatos dos profissionais de educação física, participantes desta pesquisa, referente à orientação à prática de atividade física durante a pandemia da COVID-19, Brasil $2020(\mathrm{n}=110)$.

\begin{tabular}{|c|c|}
\hline Categorias & Subcategorias - "relatos" \\
\hline Principais adaptações & $\begin{array}{l}\text { Estrutura/materiais - "Ambiente e auxiliares como tripé para celular" (Participante 4) e "Ter um celular ou notebook de qualidade, que } \\
\text { suporte a atual demanda de vídeos, imagens etc" (Participante 1) e "Tive que adaptar um ambiente que permita a aula sem exposição de } \\
\text { itens pessoais. Tive que adquirir microfone pra facilitar a compreensão para meus alunos"(Participante 30). } \\
\text { Profissionais - "Tive que comprar material para treinar/demonstrar os exercícios, assim como as alunas também; foi necessário } \\
\text { organizar melhor o tempo de planejamento das aulas, pois além de criar os treinos era necessário digitá-los no aplicativo com } \\
\text { linguagem clara, para o aluno não ter dúvidas. Foi necessário disponibilizar tempo para retirar as dúvidas dos alunos antes do treino" } \\
\text { (Participante 101) e "Gravar aulas, disponibilizar planilha, vídeo conferência para tirar dúvidas" (Participante 20) e "Necessidade de } \\
\text { aprender a lidar com as ferramentas e achar a melhor maneira de comunicação" (Participante 89). }\end{array}$ \\
\hline Pontos positivos & $\begin{array}{l}\text { Ferramentas e aplicativos - "Não perco tempo com deslocamento até o trabalho e é mais fácil de controlar grandes grupos de } \\
\text { adolescente" (Participante 5) e "Praticidade e contato frequente com clientes" (Participante 19) e "Praticidade e otimização de tempo" } \\
\text { (Participante 100) e "Otimizam tempo, é possível observar o aluno muito bem e os alunos ficam mais focados com a aula online" } \\
\text { (Participante 12). } \\
\text { Segurança - "A possibilidade de acompanhar os idosos, orientando sobre a prática adequada do exercício físico; possibilita a } \\
\text { motivação para a prática por meio de feedback e mantém a comunicação, permitindo a continuidade do cuidado" (Participante 42). } \\
\text { Profissionais - "Possibilidade de manter os pacientes ativos sem desrespeitar o isolamento social" (Participante 86). }\end{array}$ \\
\hline negativos & $\begin{array}{l}\text { Estrutura/materiais - "Não tenho disponível a estrutura de material que utilizava no clube" (Participante 5) e "Instabilidade da } \\
\text { internet" (Participante 12). } \\
\text { Ferramentas e aplicativos - "A utilização destas ferramentas é restrita de certa forma, a pessoas com um mínimo contato com } \\
\text { tecnologias. Ferramentas da Google ainda se mostram de fácil manejo, entretanto muitos dos apps e programas hoje disponíveis para } \\
\text { os profissionais não são de grande clareza para alunos de idade mais avançada e menor contato com tecnologias" (Participante 23). } \\
\text { Profissionais - "Nenhuma ferramenta substitui o presencial, temos que levar em conta alguns outros aspectos, além do físico, muitas } \\
\text { pessoas precisam do contato, do carinho, da amizade. É isso fica prejudicado" (Participante 23) e "Falta de contato com o cliente" } \\
\text { (Participante 27) e "Difícil visualização pra correções" (Participante 39). }\end{array}$ \\
\hline Feedbacks dos & $\begin{array}{l}\text { Facilidades - "Estão se adaptando e gostando, dizem que não é mesma coisa que o presencial e alguns sentem falta de equipamentos, } \\
\text { mas está suprindo as necessidades de treinos" (Participante 53) e "Surpresa positiva. Bom rendimento com os treinos online" } \\
\text { (Participante 1) e "Dizem que claro preferem aulas presenciais, porém atendem as expectativas" (Participante 13) e "Benefício de } \\
\text { conseguir treinar" (Participante 83) e "Melhora da saúde, capacidades físicas e melhora psicológica, diminuição do stress e ansiedade" } \\
\text { (Participante 69). } \\
\text { Dificuldades - "As principais dificuldades são a execução, pois o professor não está presente para corrigir os exercícios com total } \\
\text { eficácia" (Participante 2) e "Falta de motivação para treinar em casa" (Participante 3) e "Sentem falta do convívio social de algumas } \\
\text { atividades e alguns tem dificuldade em acessar treinos devido a qualidade da internet" (Participante 5) e "Alguns sentem falta de } \\
\text { equipamentos que só é possível encontrar na academia" (Participante 7) e "Dificuldade de espaço na casa deles, instabilidade da rede } \\
\text { de internet" (Participante 47). }\end{array}$ \\
\hline
\end{tabular}




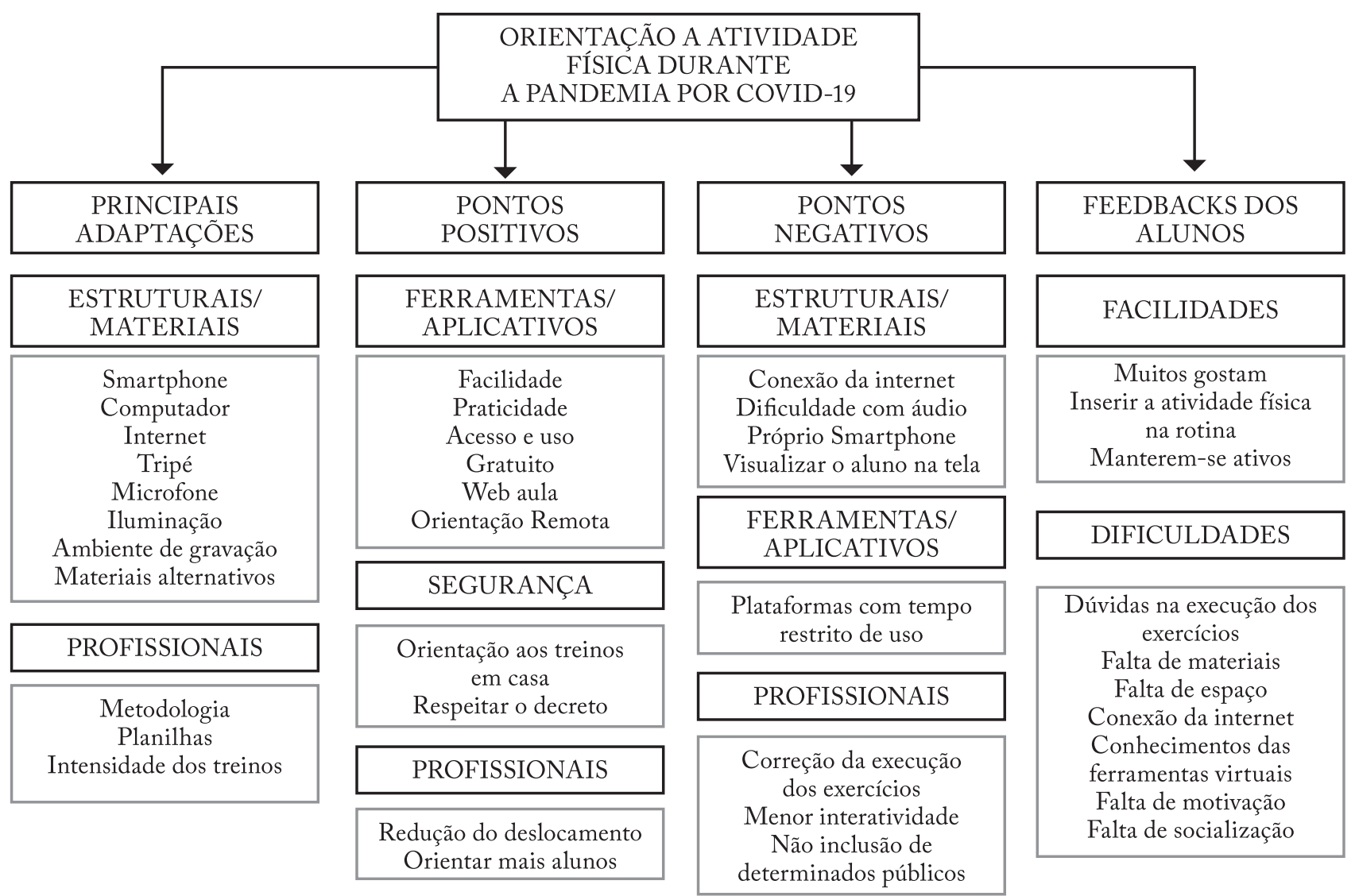

Figura 2 - Síntese das palavras mais recorrentes nos relatos dos profissionais de educação física, participantes desta pesquisa, referente à orientação à prática de atividade física durante a pandemia da COVID-19, Brasil 2020 ( $\mathrm{n}=110)$.

1) e "Telegram" $0,9 \%(n=1)$. Adicionalmente, $65,5 \%(n$ = 72) responderam que utilizam mais as ferramentas e/ ou aplicativos gratuitos.

Para além das questões optativas, os PEF puderam descrever com maiores detalhes sobre as ferramentas tecnológicas virtuais e o serviço ofertado durante a pandemia por COVID-19, sendo que os principais relatos podem ser observados abaixo no Quadro 1.

As principais adaptações realizadas pelos $\mathrm{PEF}$ foram referentes às características estruturais/materiais, como aparelho (smartphone e computador), internet, tripé, microfone, ambiente para gravar/dar aula, criar materiais alternativos e; as profissionais, como adaptar a metodologia, planilhas e intensidades dos treinos. Os profissionais também reconhecem que é necessário "melhorar a voz de comando e forma de explicar as atividades (Participante 50)" e tomar cuidado com "treinos de intensidade máxima (Participante 3)".

$\mathrm{Na}$ categoria pontos positivos, foram citados sobre as ferramentas/aplicativos incluindo a facilidade e praticidade de acesso e uso tanto por profissionais quanto para os alunos, ser gratuito (em sua maioria), ter a possibilidade de ser ao vivo (web aula) e o acompanhamen- to acontecer de forma remota. Também citaram pontos positivos sobre a segurança, pois essa seria uma forma segura de orientar o aluno em tempos de pandemia e ao mesmo tempo respeita o decreto do fechamento dos espaços públicos e academias de ginástica. Ademais, houve pontos positivos para os próprios profissionais, como a redução do tempo de deslocamento, orientar mais alunos e de diferentes cidades.

Todavia, os pontos negativos apontados foram estruturais/materiais, como a conexão instável da internet, dificuldade com o áudio, o próprio smartphone e de visualizar alguns alunos pela tela; as ferramentas/ aplicativos, como as plataformas com tempo restrito de uso e; Profissionais, como a dificuldade na correção da execução dos exercícios, redução da interatividade e a não inclusão de alguns públicos, por exemplo, o idoso. Os profissionais salientam que "nenhuma ferramenta substitui o presencial (Participante 30)".

Por fim, quanto à categoria feedbacks dos alunos que estes profissionais estão recebendo de seus alunos/ clientes, entre as facilidades identificou-se que muitos estão gostando, achando mais fácil encaixar a atividade física na rotina e tendo a possibilidade de se manterem 
ativos, mesmo durante a pandemia. Porém, alguns alunos/clientes citaram dificuldades como dúvidas quanto à execução dos exercícios, a falta de materiais e espaço adequado, conexão instável da internet e conhecimentos sobre o uso das ferramentas tecnológicas virtuais. Além disso, sentem falta de motivação e socialização e embora "alguns alunos dizem que claro, preferem aulas presenciais, porém as expectativas são atendidas (Participante 4)". Com o intuito de facilitar a visualização dos apontamentos dos PEF, foi feita uma síntese das palavras mais recorrentes nos relatos recebidos, que podem ser identificadas na Figura 2.

\section{Discussão}

Este estudo se propôs identificar as principais estratégias e ferramentas tecnológicas virtuais mais utilizadas, assim como a percepção dos PEF acerca das adaptações, pontos positivos, negativos e feedbacks dos alunos, sobre a orientação à prática de atividade física no contexto do isolamento social/restrição de circulação, em virtude da pandemia causada pelo vírus SARS-COV-2.

Dos diversos ambientes em que os PEF atendem, as academias prevalecem e esse fato advém do crescimento do mercado fitness no mundo e no Brasil, segundo o relatório global IHRSA ${ }^{17}$. Entretanto, neste ano de 2020 devido à pandemia por COVID-19 e, consequentemente ao decreto nacional 4.310/20 divulgado em março, classificando tais ambientes como "não essenciais”, os PEF ficaram limitados quanto ao local de atuação e orientação à prática de atividade física, assim como seus respectivos alunos/clientes.

Por isso, como ressaltado nos estudos de Oliveira Neto et al. ${ }^{18}$ e Chen et $\mathrm{al}^{8}$, houve um aumento na busca por treinos online em plataformas digitais, a fim de se manterem suficientemente ativos no lazer. Além de que, devido às circunstâncias impostas, os PEF ao utilizarem dos meios virtuais para continuar orientando seus alunos/clientes, os mesmos conseguem transpor as limitações impostas durante este período pandêmico, facilitando o engajamento à prática de atividade física.

Dentre as estratégias e ferramentas tecnológicas virtuais mais utilizadas pelos $\mathrm{PEF}$ estão os "vídeos gravados e disponibilizados pelo próprio profissional (60,9\%)" e o "Whatsapp web (60,0\%)", seguidas de outras ferramentas que também permitem chamadas por vídeo, como o "Zoom (43,6\%)" e outras estratégias que ofertam uma orientação por imagens e/ou escrita, como as "Planilhas no Excel/Word (37,3\%)" e aplicativos específicos como o "Mfit (29,2\%)". Desse modo, é possível notar que os PEF utilizam essas ferramentas de forma auxiliar e não substituta, pois a maioria das plataformas apontadas necessita da participação efetiva do profissional, seja na videochamada ou na organização de um planejamento de treino escrito, além de que o maior interesse é por ferramentas gratuitas.

Entretanto, assim como os participantes deste estudo bem salientaram, há pontos específicos que merecem atenção durante a orientação de forma remota, os quais estão relacionados às variáveis de prescrição de treinamento (intensidade, volume e tipos de exercícios), monitoramento do indivíduo (escalas - percepção subjetiva de esforço), basear o programa de exercícios nas recomendações já existentes (ACSM) e não deixar de lado os aspectos comportamentais (motivadores, autoeficácia, sensações do indivíduo, prazer e apoio social) ${ }^{18}$.

Dessa forma, os PEF apontaram que foi necessário realizar importantes adaptações para que o serviço fosse ofertado, principalmente estruturais/materiais, assim como a existência de pontos negativos, os quais envolvem o espaço físico, aparelhos auxiliares (smartphones e computadores) com seus acessórios (microfone, tripé e iluminação), a conexão da internet, imagem e áudio. Assim, os PEF ao ofertarem a orientação online necessitam se assegurar de que tenham ferramentas de qualidade, além de estarem preparados para adaptar o formato da aula mediante a um imprevisto caso haja problemas com a internet, como por exemplo, deixar algumas aulas gravadas, envio de um treino escrito, ter uma segunda opção de internet ou, por fim, remarcar a aula.

Também houve adaptações profissionais, quanto à metodologia e intensidade dos treinos, correção da execução dos exercícios, além das dificuldades na socialização e inclusão de diversos públicos. Nesse sentido, vale ressaltar que os idosos, faixa etária de maior risco aos efeitos adversos da COVID-19, foi o segundo público mais atendido pelos PEF participantes do presente estudo e, salientado em um dos discursos enquanto ponto negativo, os meios virtuais e consequentemente a orientação à atividade física por meio das ferramentas tecnológicas não atingem todos os tipos de alunos, por exemplo, uma grande parte de idosos.

Esse fato deve chamar atenção dos PEF, pois embora haja o declínio cognitivo, sensório motor e certa dependência na terceira idade, os idosos ainda se enxergam sem aptidão à inclusão digital, posteriormente com medo de errar, quebrar, perder ou excluir algo dos aparelhos digitais. Visto que os benefícios de incluí-los englobam a redução do isolamento, estímulo mental, 
maior socialização e uma opção de lazer, esse público prioritário necessita de estratégias que os aproximem dos meios virtuais ${ }^{19,20}$, consequentemente à qualquer tipo de serviço ofertado nesse ambiente.

Em contrapartida, também foram apontados pontos positivos como a facilidade de inserir o treino junto à nova rotina, redução do tempo de deslocamento e tanto PEF, quanto alunos/clientes manterem-se engajados na orientação e prática da atividade física, de forma segura, durante a pandemia por COVID-19. Por isso, ao considerar o meio online, as mídias e o uso do marketing digital configuram-se como um campo a ser melhor apreendido e utilizado pelos $\mathrm{PEF}^{21}$, visto que há uma relativa aceitação dos possíveis alunos/clientes na busca por personal trainer online, todavia; valorizam um atendimento mais humanizado, com propensão de bons resultados e praticidade do serviço 22 .

Além disso, os participantes deste estudo já identificam os benefícios dessa orientação remota, evidenciando a facilidade e praticidade do serviço, acesso e uso das ferramentas e plataformas digitais, além de que durante a pandemia, prezar pela segurança à saúde dos respectivos alunos/clientes é necessário. Estes, que por sua vez geram feedbacks positivos aos PEF, destacando que estão gostando da orientação, conseguem encaixar a atividade física em sua rotina com facilidade e estão mantendo-se ativos fisicamente no lazer. Esses achados salientam a importância da boa orientação profissional, mesmo que de forma remota, ponto divergente em relação aos aplicativos de treinamento programado. Embora seja crescente a busca, utilização e intenção de uso para esses aplicativos fitness ${ }^{23}$, poucos são baseados nas evidências divulgadas pelo ACSM sobre atividades aeróbias, de força e flexibilidade, reforçando assim que os usuários precisam tomar cuidado e ter atenção ao buscar por essas plataformas virtuais ${ }^{24}$.

Este estudo apresenta limitações quanto aos métodos, pois embora o convite à pesquisa tenha sido disseminado em diversos meios de comunicação virtual, pelo Conselho Nacional de Educação Física e alguns pesquisadores das cinco regiões do Brasil, houve predominância de participantes da região Sudeste e menor participação das regiões Norte e Centro-Oeste. Ademais, devido à utilização da técnica de amostragem "bola de neve", não houve como prever o número de PEF que receberam o convite à participação, resultando em não previsão de perda amostral. Acerca do instrumento desenvolvido para a coleta de dados, mesmo atingindo o objetivo pré-definido, é necessário repensar em algumas questões referentes à quantidade de alunos/clientes antes e durante a pandemia, aos impactos da pandemia na vida profissional dos PEF e realizar as coletas dos feedbacks das aulas remotas em questionário direcionado aos alunos/clientes desses PEF.

Em síntese, os PEF estão utilizando principalmente "vídeos gravados e disponibilizados pelo próprio profissional" e o "Whatsapp web", além de plataformas e aplicativos gratuitos, para orientar seus respectivos alunos/ clientes à prática de atividade física durante o contexto de isolamento social/restrição de circulação, devido à pandemia por COVID-19. Embora seja necessário realizar adaptações estruturais/materiais e profissionais, tanto $\mathrm{PEF}$ quanto seus respectivos alunos/clientes percebem a facilidade e praticidade das aulas remotas, além da possibilidade de manterem-se ativos durante a pandemia.

Por mais que a orientação à prática de atividade física de forma remota seja relativamente nova para muitos alunos/clientes, ela poderá auxiliar os PEF em suas intervenções. Embora a literatura científica apresente informações recentes e limitadas acerca dessa orientação virtual, este estudo é um dos primeiros a investigar os profissionais e suas atuações durante a pandemia por COVID-19 no Brasil. Logo, recomenda-se que futuros estudos continuem investigando o uso e o impacto das ferramentas tecnológicas virtuais enquanto facilitadora à orientação e prática de atividade física.

\section{Conflito de interesse}

Os autores declaram não haver conflito de interesses.

\section{Contribuições dos autores:}

Guimarães JAC, participou da concepção inicial do estudo, busca da literatura, redação, coleta e análise de dados, revisão crítica e finalização do estudo. Guerra PH, participou da redação, análise de dados, revisão crítica e finalização do estudo. Ueno DT, participou da busca da literatura, redação, revisão crítica e finalização do estudo. Christofoletti AEM, participou da redação, análise de dados, revisão crítica e finalização do estudo. Nakamura PM, participou da orientação e concepção inicial do estudo, redação, análise de dados, revisão crítica e finalização do estudo.

\section{Agradecimentos}

Os autores agradecem a Universidade Estadual Paulista Júlio de Mesquita Filho, Campus Rio Claro, São Paulo, ao Conselho Nacional de Desenvolvimento Científico e Tecnológico, a Coordenação de Aperfeiçoamento de Pessoal de Nível Superior e a todos que auxiliaram na divulgação e participação desta pesquisa. 


\section{Referências}

1. Worldometer. [Acessado em 2020 Ago 22]. Disponível em: https:/www.worldometers.info/coronavirus/countrieswhere-coronavirus-has-spread/.

2. World Health Organization. WHO Coronavirus Disease (COVID-19) Dashboard. [Acessado em 2020 Ago 22]. Disponível em: https://covid19.who.int/.

3. World Health Organization. Coronavirus Disease (COVID-19) pandemic. [Acessado em 2020 Mai 10]. Disponível em: https://www.who.int/emergencies/diseases/ novel-coronavirus-2019.

4. Brasil. Ministério da Saúde. Eu quero me exercitar Covid-19: como se manter saudável quando ficar em casa é a sua única opção? [Acessado em 2020 Mar 30]. Disponível em: https://saudebrasil.saude.gov.br/eu-quero-me-exercitarmais/coronavirus-como-se-manter-saudavel-quandoficarem-casa-e-a-sua-unica-opcao.

5. Carvalho FFB, Silva RG, Oliveira RB. A essencialidade das academias de ginástica para a saúde diante da pandemia da COVID-19 no Brasil. Rev Bras Ativ Fís Saúde. 2020;25:e116.

6. World Health Organization. Stay physically active during self-quarantine. [Acessado em 2020 Mar 30]. Disponível em: http://www.euro.who.int/en/health-topics/healthemergencies/coronavirus-covid19/technical-guidance/stayphysically-active-during-self-quarantine.

7. American College of Sport Medicine. Staying Physically Active During the COVID-19 Pandemic. [Acessado em 2020 Mar 20]. Disponível em: https:/www.acsm.org/readresearch/newsroom/newsreleases/newsdetail/2020/03/16/ staying-physically-active-during-covid-19-pandemic.

8. Chen P, Mao L, Nassis GP, Harmer P, Ainsworth BE, Li F. Coronavirus disease (COVID-19): The need to maintain regular physical activity while taking precautions. J Sport Health Sci. 2020;9:103-04.

9. World Health Organization. Global action plan on physical activity 2018-2030: more active people for a healthier world. Geneva: World Health Organisation; 2018. [Acessado em 2020 Abr 18]. Disponível em: https:/www.who.int/ncds/prevention/ physical-activity/global-action-plan-2018-2030/en/.

10. Ashdown-Franks G, Firth J, Carney R, Carvalho AF, Hallgren M, Koyanagi A, et al. Exercise for mental and substance use disorders: a meta-review of the benefits for neuropsychiatric and cognitive outcomes. Sports Medicine. 2020;50:151-70.

11. Nieman DC, Laurel MW. The compelling link between physical activity and the body's defense system. J Sport Health Sci. 2019;8:201-17.

12. Zhu W. Should, and how can, exercise be done during a coronavirus outbreak? An interview with Dr. Jeffrey A. Woods. J Sport Health Sci. 2020;9:105-07.
13. Spreen M. Rare populations, hidden populations and linktracing designs: what and why? Bulletin de Méthodologie Sociologique. 1992;36:34-58.

14. Faugier J, Sargeant M. Sampling hard to reach populations. J Adv Nurs. 1997;26:790-97.

15. Flick U. Introdução à pesquisa qualitativa. $3^{\mathrm{a}}$ ed. Porto Alegre: Artmed, 2009.

16. Bogdan RC, Biklen SK. Investigação qualitativa em educação: uma introdução à teoria e aos métodos. Portugal: Porto Editora, 1994.

17. Relatório Global IHRSA. Mercado mundial do fitness: principais players e mudanças no top ten. Revista ACAD Brasil, 2018. [Acessado em 2020 Ago 15]. Disponível em: https://www.acadbrasil.com.br/wp-content/uploads/2019/ 03/edicao-82.pdf.

18. Oliveira Neto L, Elsangedy HM, Tavares VDO, Teixeira CVS, Behm DG, Silva-Grigoletto ME. Traininginhome Home-based training during COVID-19 (SARS-COV2) pandemic: physical exercise and behavior-based approach. Rev Bras Fisiol Exerc. 2020;19(Suppl 2):S9-S19.

19. Farias JS, Vitor TL, Lins PV, Filho LEAP. Inclusão digital na terceira idade: um estudo sobre a propensão de idosos à adoção de tecnologias da informação e comunicação (TICs). RG\&T. 2015;15(3):164-88.

20. Andrade AM, Rabelo LN, Porto AP, Gomes EP, Lima AL. Inclusão digital na terceira idade: uma revisão de literatura. Braz. J. Hea. Rev. 2020;3(2):3231-43.

21. Silva JS, Oliveira L. O personal trainer e a sua qualificação profissional. Revista Campo do Saber. 2017;3(2):114-28.

22. Vicentini L, Santos WR, Figueiredo MJG, Morais AEA. Aceitação e Perspectivas dos Potenciais Consumidores do Serviço de Personal Trainer Online no Estado de São Paulo. VI Congresso Latino-Americano de Estudos Socioculturais do Esporte. 2018;436-453

23. García-Fernández J, Gálvez-Ruiz P, Grimaldi-Puyana M, Angosto S, Fernández-Gavira J, Bohórquez MR. The promotion of physical activity from digital services: influence of E-lifestyles on intention to use fitness app. Int. J. Environ. Res. Public Health. 2020;17(18):1-15.

24. Modave F, Bian J, Leavitt T, Bromwell J, Harris C, Vincent H. Low quality of free coaching apps with respect to the American College of Sports Medicine Guidelines: A review of current mobile apps. JMIR Mhealth Uhealth. 2015;3(3):e77.

Recebido: 28/08/2020

Aprovado: 20/10/2020 atividade física durante a COVID-19. Rev Bras Ativ Fís Saúde. 2020;25:e0150. DOI: 10.12820/rbafs.25e0150 